\title{
Pharmaco-economic analysis of adjuvant chemotherapy for stage II and III colorectal cancer
}

\author{
MINA IWAI $^{1}$, MICHIO KIMURA ${ }^{1}$, EISEKI USAMI ${ }^{1}$, TOMOAKI YOSHIMURA ${ }^{1}$ and HITOMI TERAMACHI ${ }^{2}$ \\ ${ }^{1}$ Department of Pharmacy, Ogaki Municipal Hospital, Ogaki, Gifu 503-8502; ${ }^{2}$ Laboratory \\ of Clinical Pharmacy, Gifu Pharmaceutical University, Gifu 501-1196, Japan
}

Received June 9, 2016; Accepted March 1, 2017

DOI: $10.3892 / \mathrm{mco} .2017 .1218$

\begin{abstract}
Comparison of the costs of capecitabine plus oxaliplatin (CapeOX) with that of FOLFOX6 (5-fluorouracil/leucovorin [LV] + oxaliplatin) as an adjuvant chemotherapy for stage II or III colorectal cancer has previously been reported. However, there are no reports comparing uracil and tegafur (UFT)/LV with capecitabine. Therefore, the current study compared the costs of adjuvant chemotherapy regimens including CapeOX, FOLFOX6, capecitabine and UFT/LV. The costs of chemotherapeutic drugs and for the prevention and treatment of adverse events were evaluated, as these account for the bulk of the treatment costs. Costs were expressed in Japanese Yen (US dollars). The mean costs of the chemotherapeutic drugs per patient, for an entire course of treatment, were $¥ 882,632(\$ 8,406)$ for UFT/LV, $¥ 353,290$ $(\$ 3,365)$ for capecitabine, $¥ 1,436,218(\$ 13,678)$ for FOLFOX6 and $¥ 1,255,630(\$ 11,958)$ for CapeOX. The mean costs associated with adverse events per patient were $¥ 2,210$ (\$21) for UFT/LV, $¥ 6,749$ (\$64) for capecitabine, $¥ 173,432$ (\$1,652) for FOLFOX6 and $¥ 107,430(\$ 1,023)$ for CapeOX. Therefore, the capecitabine regimen contributes to reducing costs for the management of patients with colorectal cancer who have had surgery.
\end{abstract}

\section{Introduction}

Colorectal cancer is the second most frequent malignancy and the second leading cause of cancer-associated mortality in Japan, in 2014. Over 125,000 patients are diagnosed with colorectal cancer and approximately 48,000 mortalities attributed to this neoplasm are recorded every year (1).

Correspondence to: Ms. Mina Iwai, Department of Pharmacy, Ogaki Municipal Hospital, 4-86 Minaminokawa-cho, Ogaki, Gifu 503-8502, Japan

E-mail: mina_i0913@yahoo.co.jp

Key words: adjuvant therapy, cost analysis, colorectal cancer, chemotherapy, folonic acid, fluorouracil and oxaliplatin, capecitabine and oxaliplatin, capecitabine, uracil and tegufar plus leucovorin
Postoperative provision of adjuvant chemotherapy has been a standard approach in the management of patients with colorectal cancer, in light of evidence from clinical studies indicating that it reduces the risk of tumor recurrence and mortality $(2,3)$.

The MOSAIC trial identified that, for adjuvant chemotherapy of stage II or III colon cancer, 5-fluorouracil (5-FU)/leucovorin (LV) + oxaliplatin (FOLFOX4) led to a significantly improved 5-year disease-free survival (73.3\%) and 6-year overall survival $(75.8 \%)$ than $5-\mathrm{FU} / \mathrm{LV}$ (67.4 and $76.0 \%$, respectively) (4). The subsequent NO16968 trial also found that, in patients with stage III colon cancer, capecitabine + oxaliplatin (CapeOX) produced a significantly improved 3-year disease-free survival rate $(70.9 \%)$ and 5-year overall survival rate $(77.6 \%)$ compared with 5 -FU/LV (66.5 and $74.2 \%$, respectively) (5).

The National Surgical Adjuvant Breast and Bowel Project protocol C-06 found that uracil and tegafur (UFT)/LV regimen achieved similar disease-free survival and overall survival when compared with 5-FU/LV regimen (6). Disease-free survival in the capecitabine regimen was at least equivalent to that in the 5-FU/LV regimen (7). UFT/LV and capecitabine are, therefore, an effective alternative to $5-\mathrm{FU} / \mathrm{LV}$ in the adjuvant treatment of colon cancer.

The conclusions of the clinical trials outlined above resulted in the recommendation of 5-FU/LV, CapeOX, FOLFOX, UFT/LV and capecitabine as options for the standard adjuvant chemotherapy treatment in the Japanese colon cancer treatment guidelines (8). However, despite the proven efficacy of the treatment, expenditure in drugs has recently become a great concern.

In recent years, studies of treatment cost-effectiveness found that CapeOX had superior cost-effectiveness, compared with FOLFOX6 (9-11). However, to the best of our knowledge, there have been no studies comparing UFT/LV with capecitabine.

The aim of the current study was to investigate the drug-associated costs of treatment for CapeOX as compared with FOLFOX6, and for capecitabine as compared with UFT/LV in adjuvant chemotherapy of patients with colorectal cancer, assuming equal efficacy of those treatments. The present study also evaluated whether capecitabine may become the drug regimen of choice for adjuvant chemotherapy of colorectal cancer. 
Table I. Patient characteristics.

\begin{tabular}{|c|c|c|c|c|}
\hline & $\begin{array}{c}\text { UFT+LV } \\
(n=42)\end{array}$ & $\begin{array}{l}\text { Capecitabine } \\
\quad(n=13)\end{array}$ & $\begin{array}{c}\text { FOLFOX6 } \\
(n=8)\end{array}$ & $\begin{array}{c}\text { CapeOX } \\
(\mathrm{n}=41)\end{array}$ \\
\hline Age (range), years & $72(45-83)$ & $71(51-79)$ & $66(47-72)$ & $62(41-76)$ \\
\hline Sex (male/female) & $21 / 21$ & $7 / 6$ & $6 / 2$ & $22 / 19$ \\
\hline \multicolumn{5}{|l|}{ Primary tumor site ${ }^{\mathrm{a}}$} \\
\hline Cecum & 5 & 1 & 0 & 2 \\
\hline Colon & 24 & 9 & 4 & 27 \\
\hline Rectum & 15 & 4 & 4 & 14 \\
\hline \multicolumn{5}{|l|}{ Disease stage } \\
\hline II & 12 & 3 & 0 & 1 \\
\hline IIIa & 10 & 5 & 5 & 15 \\
\hline IIIb & 16 & 4 & 2 & 21 \\
\hline IIIc & 4 & 1 & 1 & 4 \\
\hline Treatment cycles & $5(1-6)$ & $8(1-9)$ & $12(2-12)$ & $8(2-8)$ \\
\hline
\end{tabular}

aDuplications are present. UFT, uracil and tegafur; LV, leucovorin; FOLFOX6, 5-fluorouracil/leucovorin plus oxaliplatin; CapeOX, capecitabine plus oxaliplatin.

\section{Patients and methods}

Patients. Patients were identified from the medical records of the Ogaki Municipal Hospital, (Ogaki, Japan). The study was approved by the institutional Ethics Committee of the hospital. The inclusion criteria were that the patients were diagnosed with colorectal cancer stage II or III following R0 resection, and received adjuvant chemotherapy during the period between January 2013 and December 2014. Patients were excluded if they did not begin the first cycle of adjuvant chemotherapy within 2 months of radical surgery or if there were serious underlying medical conditions.

Treatments. The CapeOX regimen consisted of a 2-h intravenous infusion of $130 \mathrm{mg} / \mathrm{m}^{2}$ oxaliplatin on day 1 and $1000 \mathrm{mg} / \mathrm{m}^{2}$ oral capecitabine twice daily (morning and evening, within 30 min of last meal) on days 1 to 14 of a 3-week cycle for eight cycles. The FOLFOX6 regimen comprised a 2-h infusion of $200 \mathrm{mg} / \mathrm{m}^{2} \mathrm{LV}$ and $85 \mathrm{mg} / \mathrm{m}^{2}$ oxaliplatin, a bolus of $400 \mathrm{mg} / \mathrm{m}^{2} 5-\mathrm{FU}$, and then a $46-\mathrm{h}$ continuous infusion of $2400 \mathrm{mg} / \mathrm{m}^{2} 5$-FU repeated every 14 days for twelve cycles. Capecitabine was administered orally at a dose of $1250 \mathrm{mg} / \mathrm{m}^{2}$ twice daily, 14 days on/7 days off, for eight cycles. The UFT/LV regimen included 5-week cycle consisting of 4 weeks of oral chemotherapy followed by a 1-week rest period. UFT and LV were administered at doses of $300 \mathrm{mg} / \mathrm{m}^{2}$ and $75 \mathrm{mg}$, respectively. The daily doses of UFT and LV were divided into three doses administered $8 \mathrm{~h}$ apart and taken with water. Patients were instructed to avoid food consumption $1 \mathrm{~h}$ prior to and following each dose.

Costs. The costs of the chemotherapeutic drugs and the costs for prevention and treatment of the adverse events associated with the drug regimens were investigated. Only drug costs were investigated as they form the bulk of the costs of chemotherapy, and the costs of medical examinations and laboratory tests between regimens are similar. Cost analysis was based on the Japanese drug tariff, for the year 2016. Costs were expressed in Japanese Yen (US dollars) using the yen/dollar exchange rate of $¥ 105$ to $\$ 1$.

Statistical methods. The dose intensity was calculated as the total dose divided by the duration of dosing, while the planned dose intensity was calculated as the planned dose divided by the planned duration of dosing. The relative dose intensity (RDI) was calculated as (dose intensity/planned dose intensity)x 100.

Adverse events were evaluated according to the National Cancer Institute Common Terminology Criteria for Adverse Events Version 4.0 (12).

One-way analysis of variance was used for the age analysis of clinical characteristics. The Tukey-Kramer post hoc test was used. The $\chi^{2}$ test was used for the analysis of sex, primary tumor site and disease stage. The student's t-test was used for inter-group comparisons of treatment completion rates and costs. The Fisher's exact test was used for analyzing the incidence of adverse events. All statistical analyses were performed with EZR (v1.30, Saitama Medical Center, Jichi Medical University, Saitama, Japan), which is a graphical user interface for R (The R Foundation for Statistical Computing, Vienna, Austria) (13). $\mathrm{P}<0.05$ was considered to indicate a statistically significant difference.

\section{Results}

Patient characteristics. There were 42 patients in the UFT/LV regimen group, 13 patients in the capecitabine group, 8 patients in the FOLFOX6 group and 41 patients in the CapeOX group. The baseline characteristics were similar in the four groups (Table I). The median number of treatment cycles received was 5 (range, 1-6) in the UFT/LV group, 8 (range, 1-9) in the capecitabine group, 12 (range, 2-12) in the 
Table II. Treatment completion rate and median relative dose intensities.

\begin{tabular}{lccll}
\hline & & Treatment completion rate & & \\
& $\mathrm{N}$ & $(\%)$ & \multicolumn{2}{c}{ Relative dose intensity $(\%)$} \\
\hline UFT+LV & 42 & 73.8 & UFT & $96.2(65.4-100)$ \\
& & & LV & $99.5(69.4-100)$ \\
Capecitabine & 13 & 69.2 & Capecitabine & $92.3(70.8-100)$ \\
FOLFOX6 & 8 & 75.0 & L-OHP & $77.3(60.9-97.1)$ \\
& & & $5-F U$ & $74.0(60.9-97.1)$ \\
CapeOX & 41 & 75.6 & Capecitabine & $88.9(41.4-100)$ \\
& & & L-OHP & $86.5(36.4-100)$ \\
\hline
\end{tabular}

UFT, uracil and tegafur; LV, leucovorin; FOLFOX6, 5-fluorouracil/leucovorin plus oxaliplatin; CapeOX, capecitabine plus oxaliplatin; 5-FU, 5-fluorouracil; L-OHP, oxaliplatin.

Table III. Per-patient costs for 6 months.

\begin{tabular}{|c|c|c|c|c|c|c|}
\hline & $\mathrm{N}$ & $\begin{array}{l}\text { Chemotherapeutic } \\
\text { drugs costs of the } \\
\text { patient of } 1.5 \mathrm{~m}^{2} \\
\text { body-surface area } \\
\text { [yen (US dollars)] }\end{array}$ & $\begin{array}{c}\text { Chemotherapeutic } \\
\text { drugs costs } \\
\text { [yen } \pm \text { SD } \\
\text { (US dollars } \pm \text { SD)] }\end{array}$ & P-value & $\begin{array}{c}\text { Adverse } \\
\text { event-associated } \\
\text { related costs } \\
\text { [yen } \pm \text { SD } \\
\text { (US dollars } \pm \text { SD)] }\end{array}$ & P-value \\
\hline $\mathrm{UFT}+\mathrm{LV}$ & 42 & $\begin{array}{c}1,133,608 \\
(10,796)\end{array}$ & $\begin{array}{c}882,632 \pm 345,411 \\
(8,406 \pm 3,290)\end{array}$ & $<0.01$ & $\begin{array}{l}2,210 \pm 4,071 \\
(21 \pm 39)\end{array}$ & $<0.01$ \\
\hline Capecitabine & 13 & $\begin{array}{c}484,512 \\
(4,614)\end{array}$ & $\begin{array}{c}353,290 \pm 164,628 \\
(3,365 \pm 1,568)\end{array}$ & & $\begin{array}{c}6,749 \pm 7,669 \\
(64 \pm 73)\end{array}$ & \\
\hline FOLFOX6 & 8 & $\begin{array}{c}1,690,680 \\
(16,102)\end{array}$ & $\begin{array}{c}1,436,218 \pm 478,182 \\
(13,678 \pm 4,554)\end{array}$ & 0.36 & $\begin{array}{c}173,432 \pm 58,486 \\
(1,652 \pm 557)\end{array}$ & 0.02 \\
\hline CapeOX & 41 & $\begin{array}{c}1,467,680 \\
(13,978)\end{array}$ & $\begin{array}{c}1,255,630 \pm 515,045 \\
(11,958 \pm 4,905)\end{array}$ & & $\begin{array}{c}107,430 \pm 48,246 \\
(1,023 \pm 459)\end{array}$ & \\
\hline
\end{tabular}

The student's t-test was used to calculate P-values. UFT, uracil and tegafur; LV, leucovorin; FOLFOX6, 5-fluorouracil/leucovorin plus oxaliplatin; CapeOX, capecitabine plus oxaliplatin; SD, standard deviation.

FOLFOX6 group, and 8 (range, 2-8) in the group of patients receiving the CapeOX regimen. The recorded reasons for stopping the UFT/LV treatment were diarrhea, hepatic dysfunction and a recurrence. In the capecitabine regimen group, the reasons were hand-foot syndrome and a protraction of a hepatic dysfunction diagnosed prior to the treatment start. In one case, the patient was switched to the UFT/LV regimen after developing hand-foot syndrome. Peripheral neuropathy was the main reason reported for discontinuing treatment in the FOLFOX6 group, while diarrhea, nausea, vomiting and protracted myelosuppression were all reported in the CapeOX group. Treatment completion rates and median relative dose intensities are depicted for all groups in Table II.

Costs. The mean costs of chemotherapeutic drugs per patient, for an entire course of treatment, were ¥882,632 $(\$ 8,406)$ for UFT/LV, ¥353,290 (\$3,365) for capecitabine, ¥1,436,218 $(\$ 13,678)$ for FOLFOX6, and $¥ 1,255,630(\$ 11,958)$ for CapeOX (Table III). The mean costs associated with adverse events per patient, for an entire course of treatment, were $¥ 2,210$ (\$21) for UFT/LV, $¥ 6,749$ (\$64) for capecitabine, ¥173,432 (\$1,652) for FOLFOX6 and $¥ 107,430$ ( $\$ 1,023$ ) for CapeOX (Table III).

The chemotherapeutic drug cost of capecitabine was $40.0 \%$ lower than that of UFT/LV, per patient. The costs associated with adverse events for capecitabine were 3 times higher than those for UFT/LV. Overall, capecitabine had significantly lower costs, compared with UFT/LV $(\mathrm{P}<0.01)$.

The chemotherapeutic drug cost of CapeOX was $87.4 \%$ lower than that of FOLFOX6, per patient. In this hospital, the FOLFOX6 regimen uses innovator drugs, but generic drugs are used for LV, oxaliplatin and 5-FU. When the innovator drugs are replaced with generic drugs, the chemotherapeutic drug costs of the FOLFOX6 and CapeOX regimens per patient were $¥ 825,124(\$ 7,858)$ and $¥ 830,351(\$ 7,908)$, respectively. Therefore, there is no observable difference in the chemotherapeutic drug costs between these two drug regimens. The costs associated with adverse events for the CapeOX regimen were $61.9 \%$ lower, compared with the costs of the FOLFOX6 regimen. 


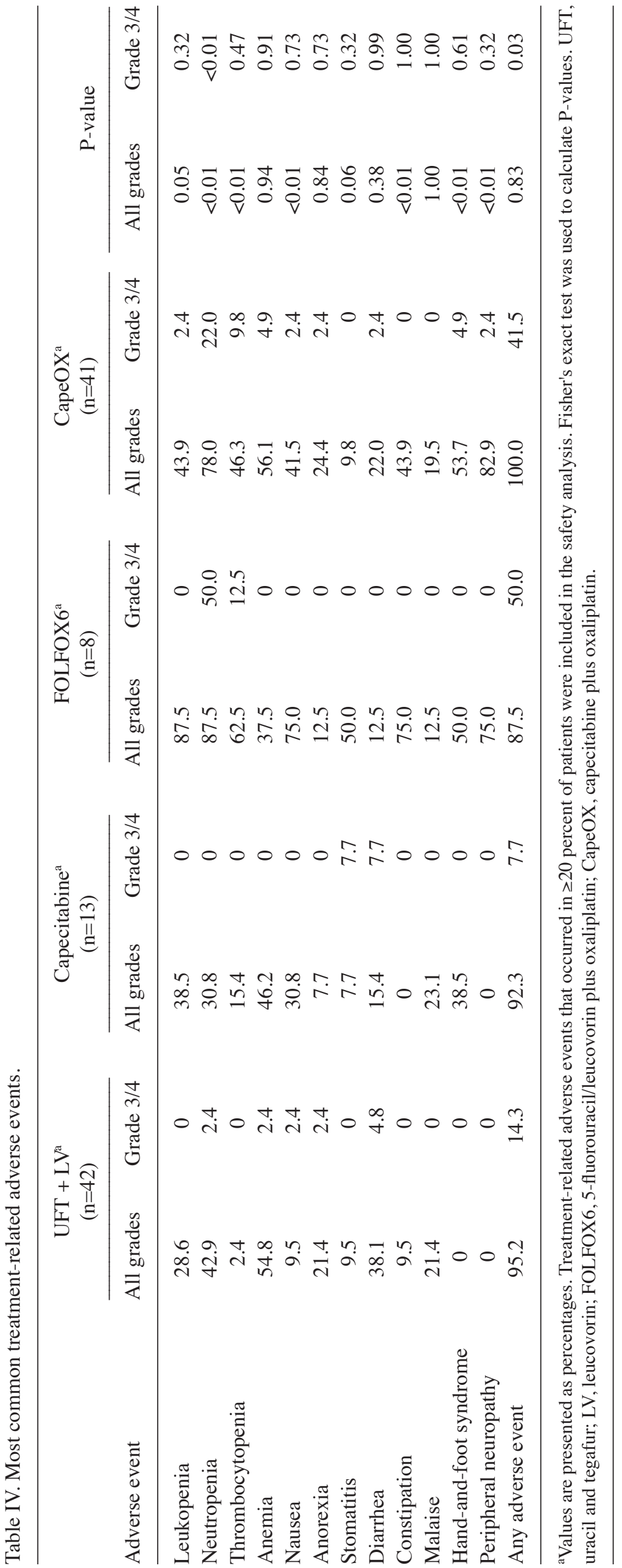


Adverse events. Treatment-related adverse events were documented in $95.2 \%$ of the patients in the UFT/LV group (grade $3 / 4,14.3 \%$ ), $92.3 \%$ of the patients in the capecitabine group (grade $3 / 4,7.7 \%$ ), $87.5 \%$ of the patients in the FOLFOX6 group (grade $3 / 4,50.0 \%$ ) and $100 \%$ of the patients in the CapeOX group (grade $3 / 4,41.5 \%$ ) (Table IV). The most frequently observed severe toxicity events were diarrhea in the UFT/LV group, and neutropenia (exceeding 20\%) in FOLFOX6 and CapeOX groups.

As anticipated with oxaliplatin, peripheral neuropathy occurred frequently in the FOLFOX6 and CapeOX groups.

\section{Discussion}

The current study was performed to assess and compare the drug-associated costs of various adjuvant chemotherapy drug regimens, namely CapeOX vs. FOLFOX6 and capecitabine vs. UFT/LV, in the therapy of colorectal cancer. In terms of adverse effect-associated costs, treatment with CapeOX costs less than treatment with FOLFOX6. However, the chemotherapeutic drug costs of the CapeOX regimen were similar to the FOLFOX6 costs. As the FOLFOX6 and CapeOX regimens may be considered equivalent in terms of treatment completion rate and RDI, and exhibit similar associated adverse events, the current study considers these two regimens to be of equal efficacy. In terms of administration, the FOLFOX6 regimen requires the insertion of a port [approximate cost $¥ 70,000$ (\$667)] and takes over $48 \mathrm{~h}$ to administer, whereas CapeOX is given as an intravenous infusion over $2.5 \mathrm{~h}$.

Xie et al (10) reported that the use of CapeOX for adjuvant chemotherapy is cost-saving, compared with FOLFOX6. Our results are in agreement with theirs when an innovator drug is used; however, the current study differs from their report when a generic drug is used instead. Results of the present study indicate that the drug-associated costs of CapeOX are equivalent to FOLFOX6 costs. It is our opinion that CapeOX should be given preference, considering that FOLFOX6 requires port insertion and on-duty h, and is associated with side effects.

In the cost comparison between capecitabine and UFT/LV, the chemotherapeutic drug costs of capecitabine were low, but its adverse event-associated costs were high. However, capecitabine had a lower incidence of grade 3 and 4 adverse events, and it is our opinion that these may be managed with supportive care. Treatment completion rates and RDIs of capecitabine and UFT/LV were similar. However, in terms of drug-associated costs, capecitabine was superior to UFT/LV.

The nature of the adjuvant chemotherapy is considered to have a role in determining treatment completion rates. No differences in treatment completion rates were observed between the drug regimens in this study. In the cases where treatment was aborted, the reason for discontinuation was non-enforcement of supportive care for the duration of the treatment. In two cases, UFT/LV and capecitabine were changed to other regimens and treatment was continued. In our opinion, treatment completion rates may be improved if supportive care is provided from the onset of the treatment. The adverse effect-associated costs of drug regimens in the present study are lower than the costs of the drugs themselves, which, in our opinion, makes adequate supportive care a cost-effective way of raising treatment completion rates. To achieve this, active pharmacist participation in supportive care is necessary.

In conclusion, drug regimens with capecitabine may contribute to reducing costs for the management of patients with colorectal cancer who have had surgery. In addition, it is necessary to provide the patients with sufficient levels of supportive care from the onset of their treatment.

\section{Acknowledgements}

The authors would like to thank Editage (www.editage.jp) for English language editing.

\section{References}

1. Foundation for promotion cancerresearch:Cancerstatistics in Japan. Available: <http://ganjoho.jp/reg_stat/statistics/brochure/backnumber/2014_jp.html>. Accessed May 14, 2016.

2. Efficacy of adjuvant fluorouracil and folinic acid in B2 colon cancer. International multicentre pooled analysis of B2 colon cancer trials (IMPACT B2) investigators. J Clin Oncol 17: 1356-1363, 1999.

3. Mamounas E, Wieand S, Wolmark N, Bear HD, Atkins JN, Song K, Jones J and Rockette H: Comparative efficacy of adjuvant chemotherapy in patients with Dukes' B versus Dukes' C colon cancer: Results from four National surgical adjuvant breast and bowel project adjuvant studies (C-01, C-02, C-03, and C-04). J Clin Oncol 17: 1349-1355, 1999.

4. André T, Boni C, Mounedji-Boudiaf L, Navarro M, Tabernero J, Hickish T, Topham C, Zaninelli M, Clingan P, Bridgewater J, et al: Oxaliplatin, fluorouracil, and leucovorin as adjuvant treatment for colon cancer. N Engl J Med 350: 2343-2351, 2004.

5. Haller DG, Tabernero J, Maroun J, de Braud F, Price T, Van Cutsem E, Hill M, Gilberg F, Rittweger K, Schmoll HJ, et al: Capecitabine plus oxaliplatin compared with fluorouracil and folinic acid as adjuvant therapy for stage III colon cancer. J Clin Oncol 29: 1465-1471, 2011.

6. Lembersky BC, Wieand HS, Petrelli NJ, O'Connell MJ, Colangelo LH, Smith RE, Seay TE, Giguere JK, Marshall ME, Jacobs AD, et al: Oral uracil and tegafur plus leucovorin compared with intravenous fluorouracil and leucovorin in stage II and III carcinoma of the colon: Results from National surgical adjuvant breast and bowel project protocol C-06. J Clin Oncol 24: 2059-2064, 2006.

7. Twelves C, Wong A, Nowacki MP, Abt M, Burris H III, Carrato A, Cassidy J, Cervantes A, Fagerberg J, Georgoulias V, et al: Capecitabine as adjuvant treatment for stage III colon cancer. $\mathrm{N}$ Engl J Med 352: 2696-2704, 2005.

8. Watanabe T, Itabashi M, Shimada Y, Tanaka S, Ito Y, Ajioka Y, Hamaguchi T, Hyodo I, Igarashi M, Ishida $\mathrm{H}$, et al: Japanese society for cancer of the colon and rectum (JSCCR) guidelines 2014 for treatment of colorectal cancer. Int J Clin Oncol 20: 207-239, 2015.

9. Wen F, Yao K, Du ZD, He XF, Zhang PF, Tang RL and Li Q: Cost-effectiveness analysis of colon cancer treatments from MOSAIC and No. 16968 trials. World J Gastroenterol 20: 17976-17984, 2014.

10. Xie Q, Wen F, Wei YQ, Deng HX and Li Q: Cost analysis of adjuvant therapy with XELOX or FOLFOX4 for colon cancer. Colorectal Dis 15: 958-962, 2013.

11. Maniadakis N, Fragoulakis V, Pectasides D and Fountzilas G: XELOX versus FOLFOX6 as an adjuvant treatment in colorectal cancer: An economic analysis. Curr Med Res Opin 25: 797-805, 2009.

12. Cancer Therapy Evaluation Program (CTEP). Common Terminology Criteria for Adverse Events (CTCAE) v4.0. Available: <http://ctep.cancer.gov/protocolDevelopment/electronic_applications/ctc.htm\#ctc_40>. Accessed March 6, 2017.

13. Kanda Y: Investigation of the freely available easy-to-use software 'EZR' for medical statistics. Bone Marrow Transplant 48: 452-458, 2013. 